\title{
WEAK BASES AND METRIZATION
}

\author{
BY
}

\author{
HAROLD W. MARTIN
}

\begin{abstract}
Several weak base (in the sense of A. V. Arhangel'skii) metrization theorems are established, including a weak base generalization of the Nagata-Smirnov Metrization Theorem.
\end{abstract}

1. Introduction. The notion of a symmetrizable space was introduced in $[4$, p. 125] by A. V. Arhangel'skii in order to study the problem of the metrization of quotient images of metrizable spaces. The idea of a weak base was then introduced in the study of symmetrizable spaces [4, p. 129]. In [10], T. Hoshina showed that every compact Hausdorff space with a point countable weak base is metrizable. This result was generalized by $\mathrm{T}$. Shiraki who showed that a regular $M^{*}$-space is metrizable if and only if it has a point countable weak base [18]. Also, R. Hodel has established a weak base generalization of the Alexandroff-Urysohn metrization theorem [9]. In $\$ 2$ we establish several weak base metrization theorems including a weak base generalization of the Nagata-Smirnov Theorem.

Let $G$ be a collection of subsets of a space $X$. If $x \in g$ for some $g \in G$, then we define $\operatorname{St}(x, G)=\bigcup\{g: x \in g \in G\}$ and $\operatorname{St}^{2}(x, G)=\bigcup\{\operatorname{St}(y, G)$ : $y \in \operatorname{St}(x, G)\}$. We assume throughout the paper that all topological spaces are $T_{1}$. Also, we let $N$ denote the set of all positive integers.

2. Weak bases and metrization. In this section we sharpen many wellknown metrization theorems, including the Nagata-Smirnov Theorem, by essentially replacing the notion of an open basis by that of a weak base.

A collection $B$ of subsets of a space $X$ is said to be a weak base for $X$ provided that to each $x \in X$, there exists $B_{x} \subset B$ such that (i) $x$ is in $b$ for every $b$ in $B_{x}$; (ii) if $a$ and $b$ are in $B_{x}$, then there exists $c$ in $B_{x}$ with $c \subset a \cap b$; (iii) a subset $F$ of $X$ is closed if and only if given $x \in X-F$, there exists $b$ in $B_{x}$ with $b$ $\cap F=\varnothing$. The concept of a weak base is due to A. V. Arhangel'skir [4, p. 129]. If to each $x \in X$ we assign a collection $B_{x}$ of supersets of $\{x\}$ such that $B=\bigcup\left\{B_{x}: x \in X\right\}$ is a weak base by virtue of the collections $B_{x}$, i.e., the

Received by the editors December 2, 1974.

AMS (MOS) subject classifications (1970). Primary 54E35.

Key words and phrases. Metrizable space, symmetrizable space, weak base, strongly uniform weak base, regular weak base, weak development. 
collections $B_{x}$ satisfy conditions (i), (ii) and (iii) of the preceding paragraph, then we say that the collection $B_{x}$ is a local weak base at $x$ for each $x$ in $X$.

Let $G_{1}, G_{2}, \ldots$ be a sequence of coverings of a space $X$ such that $G_{n+1}$ refines $G_{n}$ for all $n$; if $B_{x}=\left\{\operatorname{St}\left(x, G_{n}\right): n \in N\right\}$ is a local weak base at $x$ for each $x \in X$, i.e., if $B=\bigcup\left\{B_{x}: x \in X\right\}$ is a weak base by virtue of the collections $B_{x}$, then the sequence $G_{1}, G_{2}, \ldots$ is said to be a weak development for the space $X$. If each cover $G_{n}$ consists of open sets, then $G_{1}, G_{2}, \ldots$ is a development for $X$.

The proof of Theorem 3 of [6] carries over directly to prove the following weak base Frink Metrization Theorem. F. Slaughter has essentially established the same result and noticed, amongst others, the equivalence of Theorem 2.1 with the Niemytzki-Wilson metrization theorem [19]. With respect to the NiemytzkiWilson metrization theorem, also see [7], [8], [12] and [13].

THEOREM 2.1 (FRINK). A necessary and sufficient condition that a space $X$ be metrizable is the following. For each $x$ in $X$, there exists a decreasing sequence $\left\{V_{n}(x): n \in N\right\}=B_{x}$ of subsets of $X$ such that $B_{x}$ is a weak local base at $x$, that is, $B=\bigcup\left\{B_{x}: x \in X\right\}$ is a weak base by virtue of the collections $B_{x}$, and such that the following condition holds: given a set $V_{n}(x)$, there exists a natural number $m=m(x, n)>n$ such that if $V_{m}(x) \cap V_{m}(y) \neq \varnothing$, then $V_{m}(y) \subset V_{n}(x)$.

The 'double sequence' theorem of Nagata [16] is an easy consequence of Theorem 2.1. In fact, we have the following weak base form of this theorem.

THeOREM 2.2. A space $X$ is metrizable if and only if for each point $x$ of $X$, there exist two sequences $\left\{U_{n}(x): n \in N\right\}$ and $\left\{V_{n}(x): n \in N\right\}$ of supersets of $\{x\}$ such that the following four conditions hold: (i) for each $x$ in $X,\left\{U_{n}(x)\right.$ : $n \in N\}$ is a local weak base at $x$; (ii) given $V_{n}(x)$, there exists a natural number $m=m(x, n)$ such that $U_{m}(x) \subset V_{n}(x)$; (iii) $y$ not an element of $U_{n}(x)$ implies that $V_{n}(x) \cap V_{n}(y)=\varnothing$; (iv) $y$ in $V_{n}(x)$ implies that $V_{n}(y) \subset U_{n}(x)$.

Proof. If $\{x\}$ is an open set, then we may suppose that $U_{n}(x)=V_{n}(x)=$ $\{x\}$ for $n=1,2, \ldots$; conditions (i), (ii), (iii), and (iv) are still valid. Given $x$ in $X$ and a natural number $n$, define $H_{n}(x)=V_{1}(x) \cap \cdots \cap V_{n}(x)$.

Let $H_{n}(x)$ be given. By (ii), we have $U_{n_{i}}(x) \subset V_{i}(x)$ for $i=1,2, \ldots, n$. Let $k=\max \left\{n_{i}: i=0,1, \ldots, n\right\}$ where $n_{0}=n$. If $\{x\}$ is not open, we may choose $m$ in $N$ so that $U_{m}(x)$ is a proper subset of $\bigcap\left\{U_{i}(x): i=1,2, \ldots, k\right\}$. Then $m>k \geqslant n$ and we have $U_{m}(x) \subset H_{n}(x)$ for some $m>n$. If $\{x\}$ is open, then clearly there is $m>n$ with $U_{m}(x) \subset H_{n}(x)$. As a first consequence, it follows that $\left\{H_{i}(x): i \in N\right\}$ is a weak local base at $x$.

Let $H_{n}(x)$ be given. Choose a natural number $k>n$ such that $U_{k}(x) \subset$ $H_{n}(x)$. Choose a natural number $m>k$ such that $U_{m}(x) \subset H_{k}(x)$. We have 
$H_{m}(x) \subset U_{m}(x) \subset H_{k}(x) \subset U_{k}(x) \subset H_{n}(x)$. Suppose that $H_{m}(y) \cap H_{m}(x) \neq \varnothing$. If $y$ is not in $U_{m}(x)$, then $V_{m}(x) \cap V_{m}(y)=\varnothing$ so that $H_{m}(x) \cap H_{m}(y)=\varnothing$; it follows that $y$ is in $U_{m}(x)$. Since $U_{m}(x) \subset H_{k}(x) \subset V_{k}(x)$, we have that $y$ is in $V_{k}(x)$. It follows that $V_{k}(y) \subset U_{k}(x) \subset H_{n}(x)$. But $H_{m}(y) \subset H_{k}(y) \subset$ $V_{k}(y)$ so that $H_{m}(y) \subset H_{n}(x)$. The weak local bases $\left\{H_{n}(x): n \in N\right\}$ satisfy the conditions of Theorem 2.1, so that $X$ is metrizable, completing the proof.

J. Nagata has given an elegant proof of the Nagata-Smirnov Theorem by use of the 'double sequence' theorem [17, p. 194]; actually however, Nagata's proof established the stronger result below.

Theorem 2.3 (J. NAgAtA). A necessary and sufficient condition that a regular space $X$ be metrizable is that the following two conditions be satisfied:

(1) The space $X$ has an open basis which may be represented as a sequence $G_{1}, G_{2}, \ldots$ of closure preserving collections;

(2) $\left\{V_{n}(x): n \in N\right\}$ is a neighborhood basis for each $x$ in $X$ where $V_{n}(x)$ $=X$ if $x \notin G$ for every $G \in G_{n}$ and otherwise $V_{n}(x)=\bigcap\left\{G: x \in G \in G_{n}\right\}$.

In [5] it is shown that a regular space is metrizable if and only if it has a $\sigma$-hereditarily closure-preserving open base. An advantage of Theorem 2.3 over the classical Nagata-Smirnov Theorem may be seen in the ease with which the Burke-Engelking-Lutzer Theorem may be derived from Theorem 2.3. For the details, see [14].

We shall now establish a weak base version of Theorem 2.3.

THEOREM 2.4. A necessary and sufficient condition that a space $X$ be metrizable is that $X$ have a sequence $G_{1}, G_{2}, \ldots$ of closure-preserving covers which satisfy the following two conditions:

(1) Let $V_{n}(x)=\bigcap\left\{G: x \in G \in G_{n}\right\}$; then $\left\{V_{n}(x): n \in N\right\}$ is a local weak base at $x$ for each $x \in X$.

(2) For each $x$ in $X$ there exists $H_{i}(x)$ in $G_{i}$ such that $x$ is a member of $H_{i}(x)$ for all $i$ and such that if $V_{n}(x)$ is given, then there exists a natural number $m=m(n, x)$ for which $\operatorname{cl}\left(H_{m}(x)\right) \subset V_{n}(x)$.

Proof. Since the necessity of the condition follows easily from Stone's theorem that every metric space is paracompact [20], we need only prove the sufficiency of the condition.

If $x \in X$ and $n$ is a natural number, let $W_{n}(x)=X-\bigcup\{\operatorname{cl}(V): x \notin \operatorname{cl}(V)$ and $\left.V \in G_{n}\right\}$. If $\operatorname{cl}\left(H_{m}(x)\right) \subset V_{n}(x)$, then define $U_{n m}(x)=V_{n}(x)$ and $V_{n m}(x)$ $=H_{m}(x) \cap W_{m}(x)$; if $\mathrm{cl}\left(H_{m}(x)\right)$ is not contained in $V_{n}(x)$, then define $U_{n m}(x)$ $=X$ and $V_{n m}(x)=V_{n}(x) \cap W_{m}(x)$.

We shall show that $X$ is metrizable by showing that the sequences $\left\{U_{n m}(x)\right.$ : $n \in N ; m \in N\}$ and $\left\{V_{n m}: n \in N ; m \in N\right\}$ satisfy conditions (i), (ii), (iii) and (iv) of Theorem 2.2. 
Let $F$ be closed and $x$ be a point of $X$ not in $F$. Choose $V_{n}(x)$ with $V_{n}(x)$ $\cap F=\varnothing$. There exists a natural number $m$ with $\operatorname{cl}\left(H_{m}(x)\right) \subset V_{n}(x)$. Then $U_{n m}(x)=V_{n}(x)$ so that $U_{n m}(x) \cap F=\varnothing$. Now let $A$ be a nonempty subset of $X$ such that if $x$ is a point of $X$ not in $A$, then there exist integers $n$ and $m$, dependent upon $x$ and $A$, such that $U_{n m}(x) \cap A=\varnothing$. Since $U_{n m}(x) \cap A=\varnothing$, we have $U_{n m}(x) \neq X$. It follows that $U_{n m}(x)=V_{n}(x)$. Since $\left\{V_{n}(x): n \in N\right\}$ is a local weak base for each $x$ in $X$, it follows that $A$ is closed, completing the proof that $\left\{U_{n m}(x): n \in N ; m \in N\right\}$ is a local weak base at $x$ for each $x$ in $X$, i.e., condition (i) of Theorem 2.2 is satisfied.

Let $V_{n m}(x)$ be given. We shall show that there exist integers $j$ and $k$ such that $U_{k j}(x) \subset V_{n m}(x)$, thereby showing that condition (ii) of Theorem 2.2 is satisfied. First suppose that $V_{n m}(x)=V_{n}(x) \cap W_{m}(x)$. Since $W_{m}(x)$ is open, there exists a natural number $i$ such that $V_{i}(x) \subset W_{m}(x)$. Choose a natural number $p$ such that $V_{p}(x) \subset V_{n}(x) \cap V_{i}(x)$ and then choose a natural number $k$ such that $\operatorname{cl}\left(H_{k}(x)\right) \subset V_{p}(x)$. We have

$$
H_{k}(x) \subset V_{p}(x) \subset V_{n}(x) \cap V_{i}(x) \subset V_{n}(x) \cap W_{m}(x)=V_{n m}(x) .
$$

Since $V_{k}(x) \subset H_{k}(x)$, we have $V_{k}(x) \subset V_{n m}(x)$. Second, suppose that $V_{n m}(x)$ $=H_{m}(x) \cap W_{m}(x)$. Choose a natural number $i$ with $V_{i}(x) \subset W_{m}(x)$ and choose a natural number $k$ so that $V_{k}(x) \subset V_{i}(x) \cap V_{m}(x)$. Since $V_{m}(x) \subset H_{m}(x)$, it follows that

$$
V_{k}(x) \subset V_{i}(x) \cap V_{m}(x) \subset W_{m}(x) \cap H_{m}(x)=V_{n m}(x) .
$$

In either case, we have an integer $k$ such that $V_{k}(x) \subset V_{n m}(x)$. By condition (2), there exists an integer $j$ such that $\operatorname{cl}\left(H_{j}(x)\right) \subset V_{k}(x)$. Then, by definition, we have $U_{k j}(x)=V_{k}(x)$. It follows that $U_{k j}(x) \subset V_{n m}(x)$, as desired.

Assume that $y$ is not a point of $U_{n m}(x)$. Then $U_{n m}(x) \neq X$ whence $V_{n m}(x)=H_{n}(x) \cap W_{m}(x)$ and $\operatorname{cl}\left(H_{m}(x)\right) \subset V_{n}(x)=U_{n m}(x)$ so that $y$ is not in $\operatorname{cl}\left(H_{m}(x)\right)$. Since $y$ is not in $\operatorname{cl}\left(H_{m}(x)\right)$, we have $\operatorname{cl}\left(H_{m}(x)\right) \cap W_{m}(y)=\varnothing$. Since $V_{n m}(x) \subset H_{m}(x)$ and $V_{n m}(y) \subset W_{m}(y)$, it follows that $V_{n m}(x) \cap$ $V_{n m}(y)=\varnothing$ showing that condition (iii) of Theorem 2.2 is satisfied.

Suppose that $y$ is an element of $V_{n m}(x)$. Since $V_{n m}(x) \subset V_{n}(x)$, we have that $y$ is also an element of $V_{n}(x)$ so that $V_{n}(y) \subset V_{n}(x)$. But $V_{n m}(y) \subset$ $V_{n}(y)$ so that $V_{n m}(y) \subset V_{n}(x) \subset U_{n m}(x)$, that is, condition (iv) of Theorem 2.2 is satisfied. The metrizability of $X$ now follows by Theorem 2.2, completing the proof.

We shall now prove a weak base version of Morita's 'double starring' metrization theorem [15]. In order to avoid multiple subscripts in the proof of Theorem 2.5, we shall write sequences in functional notation; thus, we write $x(n)$ instead of $x_{n}$ and $x(n(i))$ instead of $x_{n_{i}}$, etc. 
THEOREM 2.5. A necessary and sufficient condition that a topological space $X$ be metrizable is that $X$ have a weak development $G(1), G(2), \ldots$ such that if $B_{x}=\left\{\mathrm{St}^{2}(x, G(n)): n \in N\right\}$ for each $x \in X$, then $B=\bigcup\left\{B_{x}: x \in X\right\}$ is a weak base by virtue of the collections $B_{x}$.

Proof. The necessity of the condition is clear. To prove the sufficiency, let $G(1), G(2), \ldots$ be a weak development such that $B=\bigcup\left\{B_{x}: x \in X\right\}$ is a weak base by virtue of the collections $B_{x}=\left\{\operatorname{St}^{2}(x, G(n)): n \in N\right\}$.

We shall first show that if a sequence $\{x(n)\}$ converges to $x$, then given any natural number $m,\{x(n)\}$ is eventually in the set $\operatorname{St}(x, G(m))$. Let $\{x(n)\}$ converge to $x$ and define $S=\{x\} \cup\{x(n): n \in N\}$. Suppose that $S$ is not closed. Then there exists a point $y$ not in $S$ such that $\operatorname{St}(y, G(n)) \cap S \neq \varnothing$ for all natural numbers $n$; that is, there exists a subsequence $\{x(i(n))\}$ of $\{x(n)\}$ with $x(i(n))$ in $\operatorname{St}(y, G(n))$ for each natural number $n$. Now let $A=\{y\} \cup\{x(i(n))$ : $n \in N\}$ and suppose that $A$ is not closed. Then there exists a point $z$ not in $A$ such that $\operatorname{St}(z, G(n)) \cap A \neq \varnothing$ for all natural numbers $n$; that is, $x(i(n(j))) \in$ $\operatorname{St}(z, G(j))$ for $j \in N$. We also have $x(i(n(j)))$ in the set $\operatorname{St}(y, G(n(j)))$ for all $j \in N$. Since $n(j)>j$, it follows that $\operatorname{St}(y, G(n(j))) \subset \operatorname{St}(y, G(j))$ so that $\operatorname{St}(z, G(j)) \cap \operatorname{St}(y, G(j)) \neq \varnothing$ for all $j \in N$. Since $z$ and $y$ are distinct, this is clearly impossible and necessarily the set $A$ is closed. But $\{x(i(n))\}$ converges to $x$ and $x$ is not in $A$, so that the set $A$ cannot be closed; this contradiction shows that the set $S$ must be closed.

Now suppose that there exists a natural number $m$ and a subsequence $\{x(n(i))\}$ of $\{x(n)\}$ such that $x(n(i))$ is not an element of St $(x, G(m))$ for each natural number $i$, that is, suppose that $\{x(n)\}$ is not eventually in the set St $(x, G(m))$ for some $m$. Since $\{x(n)\}$ converges to $x$, necessarily $\{x(n(i))\}$ converges to $x$. By the argument of the previous paragraph, the set $B=\{x\} \cup$ $\{x(n(i)): i \in N\}$ is closed. But $x(n(i))$ not being in $\operatorname{St}(x, G(m))$ for all $i \in N$ then implies that the set $B-\{x\}$ is also closed, which contradicts the fact that $\{x(n(i))\}$ converges to $x$. It follows that the sequence $\{x(n)\}$ is eventually in the set St $(x, G(m))$ for any natural number $m$, that is, $\{x(n)\}$ converges to $x$ if and only if $\{x(n)\}$ is eventually in every set $\operatorname{St}(x, G(m)), m \in N$.

We shall now show that the space $X$ is first countable. Given a point $x$ in $X$ and a natural number $n$, let $H=X-\operatorname{St}^{2}(x, G(n))$ and let $F=\left\{y: \operatorname{St}^{2}(y, G(i))\right.$ $\cap H \neq \varnothing$ for all $i \in N$ \}. Assume that $F$ is not closed. Then there is a point $z$ not in $F$ with $\operatorname{St}(z, G(i)) \cap F \neq \varnothing$ for each natural number $i$, that is, there exists a sequence $\{f(i)\}$ in $F$ with $f(i) \in \operatorname{St}(z, G(i))$ for all $i \in N$. Given a definite $f(i)$, there exists a sequence $\{h(j)\}$ in $H$ such that $h(j)$ is in $\operatorname{St}^{2}(f(i), G(j))$ for each natural number $j$. Then $\{h(j)\}$ converges to $f(i)$ so that $\{h(j)\}$ is eventually in any set $\operatorname{St}(f(i), G(k))$ for each natural number $k$. Thus there exists a subse- 
quence $\{h(j(k))\}$ of $\{h(j)\}$ such that $h(j(k))$ is in $\operatorname{St}(f(i), G(k))$ for all $k \in N$. In particular, we have $h(j(i))$ in $\operatorname{St}(f(i), G(i))$. Since $f(i)$ is an element of St $(z, G(i))$ for all natural numbers $i$, we have $h(j(i)) \operatorname{int}\left(\operatorname{St}^{2}(z, G(i))\right.$ for all $i \in N$. But then, by the definition of $F$, we have $z$ in $F$, which contradiction shows that the set $F$ must be closed. It follows that $x \in \operatorname{int}\left(\operatorname{St}^{2}(x, G(n))\right)$, that is, the space $X$ is first countable.

Suppose that $x$ is not in int $(\operatorname{St}(x, G(n)))$. Then, since $X$ is first countable, there exists a sequence $\{a(i)\}$ in $X-\operatorname{St}(x, G(n))$ with $\{a(i)\}$ convergent to $x$. It follows that there exists a subsequence $\{a(i(j))\}$ of $\{a(i)\}$ with $a(i(j)) \in$ $\operatorname{St}(x, G(j))$ for all $j \in N$, which is a contradiction. It follows that $x$ is in $\operatorname{int}(\operatorname{St}(x, G(n)))$ for all $x$ in $X$ and all natural numbers $n$.

For each $x$ in $X$ and natural number $n$, let $V_{n}(x)=\operatorname{St}(x, G(n))$. Given a set $V_{n}(x)$, since $x$ is in int $\left(V_{n}(x)\right)$, there exists a natural number $m>n$ such that $\operatorname{St}^{2}(x, G(m)) \subset V_{n}(x)$; similarly, there exists a natural number $k>m$ such that $\operatorname{St}^{2}(x, G(k)) \subset V_{m}(x)$. If $V_{k}(x) \cap V_{k}(y) \neq \varnothing$, then it is easy to show that $V_{k}(y) \subset V_{n}(x)$. That $X$ is metrizable now follows by Theorem 2.1, completing the proof.

The following theorem may now be established from Theorem 2.5.

THEOREM 2.6. The following are equivalent for a space $X$.

(1) The space $X$ is metrizable.

(2) The space $X$ has a weak development $G_{1}, G_{2}, \ldots$ such that if $x \in V$ where $V$ is open, then there exists a neighborhood $U$ of $x$ and a natural number $n$ for which $\operatorname{St}\left(U, G_{n}\right) \subset V$.

(3) The space $X$ has a weak development $G_{1}, G_{2}, \ldots$ such that if $A \subset V$ where $A$ is compact and $V$ is open, then $\operatorname{St}\left(A, G_{n}\right) \subset V$ for some $n$.

Proof. That (1) implies (2) and (2) implies (3) are obvious. Therefore, assume that $G_{1}, G_{2}, \ldots$ is a weak development which satisfies condition (3). Suppose that $G_{1}, G_{2}, \ldots$ does not satisfy the double starring condition of Theorem 2.5. Then there exists $x$ in $V$ where $V$ is open such that $\operatorname{St}^{2}\left(x, G_{n}\right)$ is not contained in $V$ for all $n \in N$. For each natural number $n$, there exists $H_{n}$ in $G_{n}$ such that $\operatorname{St}\left(x, G_{n}\right) \cap H_{n} \neq \varnothing$ and $H_{n}$ is not a subset of $V$. Let $x_{n}$ be an element of $\operatorname{St}\left(x, G_{n}\right) \cap H_{n}$ for all $n \in N$. There exists a natural number $M$ such that $x_{n} \in$ $V$ for all $n>M$. Let $S=\{x\} \cup\left\{x_{n}: n>M\right\}$. We have $S \subset V$ and $S$ is compact. By condition (3), we must have $\operatorname{St}\left(S, G_{m}\right) \subset V$ for some $m \in N$, which is a contradiction since $H_{m} \subset \operatorname{St}\left(S, G_{m}\right)$. It follows that $G_{1}, G_{2}, \ldots$ must satisfy the double starring condition of Theorem 2.5 , completing the proof.

The antecedents of Theorem 2.6 are as follows: F. B. Jones showed that a regular space is metrizable if and only if it has a development satisfying condition (3) of Theorem 2.6 [11]; A. V. Arhangel'skĩ [2] and A. H. Stone [21] showed 
that a $T_{0}$-space is metrizable if and only if it has a development which satisfies condition (2) of Theorem 2.6.

A weak base $B$ for a space $X$ is said to be strongly uniform provided that if $K \subset V$ where $K$ is compact and $V$ is open, then only finitely many members of $B$ simultaneously meet both $K$ and $X-V$. In [3], Arhangel'skii showed that a Hausdorff space is metrizable if and only if it has a strongly uniform open base. This theorem, for weak bases, is an easy consequence of Theorem 2.6.

THEOREM 2.7. A topological space $X$ is metrizable if and only if $X$ has a strongly uniform weak base.

Proof. Let $B=\left\{B_{x}: x \in X\right\}$ be a strongly uniform weak basis for the space $X$, where $B_{x}$ is a local weak base at $x$ for each $x$ in $X$. If $x$ is an element of $X$ such that $\{x\}$ is open, then let $V_{n}(x)=\{x\}$ for each natural number $n$. If $\{x\}$ is not open, let $V_{n}(x)$ be the intersection of $n$ distinct elements of $B_{x}$. Let $G_{n}=\left\{V_{n}(x): x \in X\right\}$. The sequence $G_{1}, G_{2}, \ldots$ is easily seen to be a weak development for $X$. Let $A \subset V$ where $A$ is compact and $V$ is open. Since $B$ is strongly uniform, only finitely many members of $B$ meet both $A$ and $X-V$, say $m$ members of $B$. Then $\operatorname{St}\left(A, G_{m+1}\right) \subset V$, for if this is not true, then there exists $H=V_{m+1}(x) \in G_{m+1}$ such that $H \cap A \neq \varnothing$ and $H \cap(X-V) \neq \varnothing$. But $V_{1}(x), V_{2}(x), \ldots, V_{m+1}(x)$ are $m+1$ distinct members of $B$, each of which meets both $A$ and $X-V$, which is a contradiction. Consequently, $\operatorname{St}\left(A, G_{m+1}\right) \subset V$ and $X$ is metrizable by Theorem 2.6. The converse follows easily from A. H. Stone's theorem that every metric space is paracompact, completing the proof.

A weak base $B$ for a space $X$ is said to be regular provided that if $x \in V$ where $V$ is open, then there exists an open set $U$ such that $x \in U \subset V$ and only finitely many members of $B$ simultaneously meet both $U$ and $X-V$. In [1] Arhangel'skii showed that a topological space $X$ is metrizable if and only if it has a regular open basis. The following is almost immediate from Theorem 2.7.

Theorem 2.8. A topological space $X$ is metrizable if and only if $X$ has a regular weak basis.

\section{REFERENCES}

1. A. V. Archangel'skii, On the metrization of topological spaces, Bull. Acad. Polon. Sci. Sér. Sci. Math. Astronom. Phys. 8 (1960), 589-595. (Russian) MR 23 \#A2861.

2. - New criteria for paracompactness and metrisability of an arbitrary $T_{1^{-}}$. space, Dokl. Akad. Nauk SSSR 141 (1961), 13-15 = Soviet Math. Dokl. 2 (1961), 13671369. MR 24 \#A1113.

3. - Bicompact sets and the topology of spaces, Dokl. Akad. Nauk SSSR 150 (1963), 9-12 = Soviet Math. Dokl. 4 (1963), 561-564. MR 27 \#720; 28 p. 1246.

4. - Mappings and spaces, Uspehi Mat. Nauk 21 (1966), no. 4 (130), 133-184 = Russian Math. Surveys 21 (1966), no. 4, 115-162. MR 37 \#3534. 
5. D. Burke, R. Engelking and D. Lutzer, Hereditarily closure-preserving collections and metrization, Proc. Amer. Math. Soc. 51 (1975), 483-488.

6. A. H. Frink, Distance functions and the metrization problem, Bull. Amer. Math. Soc. 43 (1937), 133-142.

7. P. W. Harley, Metric and symmetric spaces, Proc. Amer. Math. Soc. 43 (1974), 428-430.

8. P. W. Harley and G. D. Faulkner, Metrization of symmetric spaces (to appear).

9. R. E. Hodel, Metrizability of topological spaces (to appear).

10. T. Hoshina, On the quotient s-images of metric spaces, Sci. Rep. Tokyo Kyoiku Daigaku Sect. A 10 (1970), 265-268. MR 43 \#1115.

11. F. B. Jones, Metrization, Amer. Math. Monthly 73 (1966), 571-576. MR 33 \#7980.

12. H. W. Martin, Metrization of symmetric spaces and regular maps, Proc. Amer. Math. Soc. 35 (1972), 269-274. MR 46 \#2648.

13. - Perfect maps of symmetrizable spaces, Proc. Amer. Math. Soc. 38 (1973), 410-412. MR 47 \#2561.

14. - A note on the Frink metrization theorem, Rocky Mountain J. Math. 6 (1976), 155-157.

15. K. Morita, A condition for the metrizability of topological spaces and for n-dimensionality, Sci. Rep. Tokyo Kyoiku Daigaku Sect. A 5 (1955), 33-36. MR 17, 179.

16. J. Nagata, $A$ contribution to the theory of metrization, J. Inst. Polytech. Osaka City Univ. Ser. A 8 (1957), 185-192. MR 20 \#4256.

17. - Modern general topology, Bibliotheca Mathematica, vol. 7, North-Holland, Amsterdam; Wolters-Noordhoff, Groningen, 1968. MR 41 \#9171.

18. T. Shiraki, On some metrization theorems (to appear).

19. F. G. Slaughter, Jr., Theorems on submetrizability (unpublished).

20. A. H. Stone, Paracompactness and product spaces, Bull. Amer. Math. Soc. 54 (1948),977-982. MR 10, 204.

21. - Sequences of coverings, Pacific J. Math. 10 (1960), 689-691. MR 22 \#9955.

DEPARTMENT OF MATHEMATICS, COLLEGE OF ST. SCHOLASTICA, DULUTH, MINNESOTA 55811 